\title{
O.S.P.
}

\section{Rapport au savoir et construction des parcours de formation au sein de la formation professionnelle initiale de niveau $\mathrm{V}$}

Valérie Capdevielle-Mougnibas, Yves Prêteur et Cécile Favreau

\section{(2) OpenEdition \\ Journals}

Édition électronique

URL : http://journals.openedition.org/osp/4056

DOI : 10.4000/osp.4056

ISSN : 2104-3795

Éditeur

Institut national d'étude du travail et d'orientation professionnelle (INETOP)

Édition imprimée

Date de publication : 7 mars 2013

ISSN : 0249-6739

Référence électronique

Valérie Capdevielle-Mougnibas, Yves Prêteur et Cécile Favreau, « Rapport au savoir et construction des parcours de formation au sein de la formation professionnelle initiale de niveau $V$ », L'orientation scolaire et professionnelle [En ligne], 42/1 | 2013, mis en ligne le 07 mars 2016, consulté le 30 avril 2019. URL : http://journals.openedition.org/osp/4056 ; DOI : 10.4000/osp.4056

Ce document a été généré automatiquement le 30 avril 2019.

(c) Tous droits réservés 


\title{
Rapport au savoir et construction des parcours de formation au sein de la formation professionnelle initiale de niveau $V$
}

\author{
Valérie Capdevielle-Mougnibas, Yves Prêteur et Cécile Favreau
}

\section{Introduction}

1 La lutte contre les sorties du système éducatif sans qualification constitue une priorité politique européenne, qui avait déjà été clairement affirmée en France par la loi d'orientation sur l'éducation de 1989 puis la loi quinquennale sur le travail, l'emploi et la formation professionnelle de 1993. Pourtant, après une baisse spectaculaire au cours des décennies précédentes, le nombre de jeunes non titulaires d'un Certificat d'Aptitudes Professionnelles (CAP), Brevet d'Études Professionnelles (BEP) ou d'un baccalauréat s'est stabilisé depuis le début des années quatre-vingt-dix ${ }^{1}$ (Léger, 2008). En France, les sorties du système scolaire sans certification reconnue représentent environ $17 \%$ de la totalité des sortants de formation initiale soit entre 110000 et 170000 jeunes selon le mode de calcul retenu (Joseph, Lopez, \& Ryk, 2008). Parmi eux, tous ne sont pas pour autant sans qualification. D'un point de vue restrictif et selon la définition française, moins de $6 \%$ des jeunes ayant arrêté pour la première fois de se former après une classe de premier cycle (collège) ou une première année de CAP ou de BEP quittent véritablement les études sans diplôme et sans aucune qualification (MEN-DEPP, 2008).

Ces jeunes dits «sans qualification » ont majoritairement connu des difficultés scolaires, sont issus de milieux défavorisés et sont souvent d'origine étrangère (Langouët, 2003). Ils constituent une population hétérogène. Parmi les sortants qui en 2005 ont arrêté leur scolarité, $35 \%$ sont issus du collège ou de la première année de CAP-BEP, $24 \%$ ont abandonné en dernière année d'un CAP-BEP, $12 \%$ après une seconde ou première 
générale ou technologique et $29 \%$ jusqu'en dernière année de préparation du baccalauréat (Léger, 2008). Même si ce phénomène ne spécifie pas seulement cette filière, la partie la plus importante des sortants sans qualification est issue des filières de la formation professionnelle initiale de niveau V. En 2005, elle représentait environ $60 \%$ des arrêts de scolarité avant la fin du cycle secondaire contre $40 \%$ pour les autres filières (Léger, 2008; MEN-DEPP, 2008). Cette contribution conséquente impose de réfléchir aux conditions de réussite d'un parcours de formation au sein de la formation professionnelle initiale. Quels sont les facteurs explicatifs des sorties anticipées en lycée professionnel et en CFA ? Les mêmes processus sont-ils à l'œuvre dans les deux systèmes de formation? Les caractéristiques du parcours scolaire antérieur jouent-elles un rôle dans le devenir au sein de la formation professionnelle initiale?

\section{Expliquer les sorties sans qualification : les enjeux d'une controverse}

3 Si l'on connaît mieux aujourd'hui certaines spécificités des jeunes sans qualification tant du point de vue de leurs conditions d'entrée dans la vie active que de leurs origines scolaires et familiales, il apparaît plus compliqué d'expliquer la persistance et l'ampleur du phénomène des sorties sans qualification qui dépend non seulement de la variation des effectifs concernés (effet démographique) mais aussi et surtout de l'échec de ces publics aux examens et de l'abandon en cours de formation (effet scolarisation).

Les recherches centrées sur cette question s'inscrivent dans une controverse qui crée les conditions d'une rencontre entre la sociologie et la psychologie de l'éducation au risque de perdre de vue la frontière séparant les deux champs disciplinaires. Dans les deux disciplines, les deux phénomènes d'échec et d'abandon sont souvent appréhendés à partir d'une approche pragmatique des facteurs discriminants (filières de scolarisation, caractéristiques sociofamiliales, âge, genre, niveau scolaire, etc.), inscrite dans une perspective post-positiviste (Guba \& Lincoln, 1994) visant à repérer des indicateurs de risque souvent hiérarchisés et proposant des typologies des publics à risque (Broccolichi \& Largueze, 1996 ; Caille, 2000 ; Fortin, Royer, Potvin, Marcotte, \& Yergeau, 2004 ; Gagné \& Marcotte, 2010 ; Langevin, 1999 ; Lessard, Fortin, Joly, Royer, Marcotte, \& Potvin, 2007). Ces approches descriptives tendent à proposer une vision fragmentée, cristallisée sur quelques facteurs envisagés à partir d'un schéma causal objectiviste, linéaire et univoque. Elles privilégient souvent une explication où les phénomènes susceptibles de générer une sortie sans qualification sont envisagés comme la conséquence des caractéristiques psychologiques de l'élève qui abandonne (défaut de structuration du projet professionnel, niveau de compétences cognitives, etc.).

Ces approches statistiques se différencient des travaux inscrits dans un paradigme épistémologique constructiviste tel que défini par Guba et Lincoln (1994) dont l'opérationnalisation vise à privilégier le point de vue de l'acteur en développement (Capdevielle-Mougnibas \& de Léonardis, 2010 ; Charlot, Emin, \& Jellab, 2002 ; CourtinatCamps \& Prêteur, 2012 ; Esterle-Hedibel, 2007 ; Lamamra \& Masdonati, 2009; Rochex, 1998). Ces recherches qualitatives insistent ainsi sur le fait que l'abandon comme l'échec à l'examen doivent être analysés comme des processus complexes, susceptibles de prendre des formes diverses ${ }^{2}$ qui ne peuvent être dissociés des caractéristiques des contextes de formation et des savoirs qui y sont enseignés ainsi que de l'expérience sociale et 
subjective des élèves concernés. Ils montrent ainsi la nécessité de prendre en compte le sens de l'expérience scolaire selon les rapports dialectiques qui se tissent entre un sujet particulier et des autruis significatifs, relevant des différents milieux de vie et institutions, historiquement situés, auxquels il participe.

\section{Objectifs et stratégie de recherche}

6 Notre contribution présente une partie des résultats d'une recherche ${ }^{3}$ comparative (170 lycéen(ne)s professionnel(le)s, 441 apprenti(e)s salarié(e)s), longitudinale (deux ans)) visant à repérer et analyser les processus déterminants de la construction des parcours de formation (abandon/poursuite de la formation - réussite/échec au diplôme) au sein de la formation professionnelle initiale. Il s'agira plus particulièrement d'envisager les conséquences des aléas du parcours scolaire antérieur sur la construction des trajectoires de formation au sein de chacune des deux filières de formation professionnelle (lycée professionnel et apprentissage).

7 Nos travaux, inscrits dans une approche développementale, constructionniste (Von Glasersferld, 1990) donnent priorité au social et considèrent la réalité comme coconstruite dans l'expérience avec les autres (Malrieu, 1978, 1995). Ils appréhendent les conditions de réussite dans la formation professionnelle initiale de niveau $\mathrm{V}$ à partir d'une approche relationnelle et contextuelle de la production des inégalités et plus largement des réalités scolaires (Capdevielle-Mougnibas, 2010; Capdevielle-Mougnibas, Garric, Courtinat-Camps, \& Favreau, 2012 ; Capdevielle-Mougnibas, Prêteur, \& RossiNeves, 2008; de Léonardis, Capdevielle-Mougnibas, \& Prêteur, 2006; Favreau \& Capdevielle-Mougnibas, 2011). Nous mettons l'accent sur la problématique du rapport au savoir qui, à partir d'une perspective interactionniste, permet de dépasser une explication se limitant aux seuls éléments objectifs ou au contraire subjectifs pour expliquer les avatars de la scolarité (Laterrasse \& Brossais, 2006). Le rapport au savoir est envisagé comme une forme de rapport au monde qui renvoie à «un ensemble de jugements évaluatifs et d'attitudes [...] portant à la fois sur ce qui vaut d'être appris, sur ce qui est légitime, pertinent et utile de savoir, et sur la signification même accordée aux termes tels que savoir, apprendre et aux activités et compétences qu'ils désignent selon les individus ou les groupes sociaux » (Rochex, 1995, p. 128). La posture théorique que nous adoptons, consiste à étudier les modes de socialisation familiale et scolaire dans un rapport dialectique avec les processus de personnalisation et de subjectivation (Malrieu, 2003). Il s'agit principalement d'étudier les mobiles de l'activité en dégageant le sens que les élèves de la formation professionnelle initiale accordent à leur expérience d'apprenants en fonction de leur histoire sociale, familiale et scolaire. Une des principales originalités de nos recherches qualitatives réside dans le choix de méthodologies mixtes qui nous conduit à privilégier l'utilisation de questionnaires pour étudier le sens que les acteurs accordent au savoir et aux activités qui lui sont attachées. Nous opérationnalisons nos recherches en privilégiant l'analyse du sens de l'expérience scolaire (Rochex, 1995) et du rapport à l'apprendre (Charlot, 1999) considérés dans notre perspective comme des composantes du rapport au savoir.

8 Dans cette recherche, nous nous sommes plus particulièrement attachés à analyser les incidences du parcours et des formes de rapport au savoir construites par les élèves à l'école primaire et au collège sur la réussite des parcours de formation au sein de l'enseignement professionnel. Nous faisons l'hypothèse que, pour les apprentis et les 
lycéens professionnels de niveau $\mathrm{V}$, au-delà de l'influence des caractéristiques objectives du parcours scolaire, le sens de l'expérience scolaire et le rapport à l'apprendre ont un rôle dans la genèse aussi bien de l'abandon de formation que de l'échec à l'examen.

\section{Participants}

9 L'échantillon est constitué de 641 sujets préparant 9 CAP enseignés à la fois en lycée et en Centre de formation pour apprentis (CFA) que nous avons catégorisés de nouveau en 3 groupes: Services à la personne (coiffure, esthétique); Bâtiment (charpente, menuiserie, ébénisterie, peinture, maçonnerie, plomberie); Mécanique automobile. Le nombre d'apprentis par spécialité dépend de l'offre de formation de chaque établissement participant. Nous avons rencontré systématiquement la totalité des jeunes inscrits en première année dans les filières retenues. La population se compose de 170 lycéen(ne)s professionnel(le)s et 441 apprenti(e)s et 462 garçons (soit $76 \%$ de l'échantillon global) et 149 filles (soit $24 \%$ ), âgé(e)s de 15 à 19 ans (âge moyen de 16 ans 8 mois [écart-type 1,87] inscrit(e)s en $1^{\text {re }}$ année de CAP/ BEP dans 8 établissements de la région Midi-Pyrénées (4 lycées professionnels : A, B, C, D - 4 Centres de Formation d'Apprentis (CFA) : E, F, G, H).

Conformément à la littérature, nos résultats révèlent que la formation professionnelle initiale valide toujours les stéréotypes de sexe les plus traditionnels. Filles et garçons y fréquentent des lieux, des filières et des sections de formations très différents ${ }^{4}$ (Capdevielle-Mougnibas, 2010). De la même manière, lycées et CFA n'accueillent pas les mêmes publics d'élèves. Les lycéens apparaissent plus jeunes que leurs homologues ( $T$ $(5.97)=35.67, p<.001)$. Les apprentis ont ainsi en moyenne un an de moins que les lycéens qui constituent une population plus homogène (17 ans 1 mois [écart-type 2.02] contre 16 ans 1 mois [écart-type 1.11]). Ces résultats généraux doivent néanmoins être nuancés en fonction de plusieurs sous-catégories dont certaines apparaissent plus représentées que d'autres. $75 \%$ des lycéens avaient 16 ans ou moins contre $63 \%$ des apprentis au moment de la rencontre avec le chercheur $\left(\mathrm{X}^{2}(3)=49.10, p<.0001\right)$. La catégorie des 18 ans et + est ainsi très peu représentée chez les lycéens.

11 Pour caractériser le «capital scolaire » et socioprofessionnel de la famille, nous avons construit un indicateur global. Cette cote sociale combine trois étapes: la première articule les niveaux d'études des deux parents, la deuxième regroupe de façon similaire les professions des deux parents, enfin la troisième étape articule les deux indicateurs : niveaux d'études et professions. Ce critère synthétique évite à la fois de multiplier le nombre des calculs pour chacun des quatre indicateurs (niveau d'études et profession de chacun des parents) et surtout, il traduit un ensemble de ressources au sein de chaque famille considérée comme un groupe familial gérant à sa manière ce patrimoine socioéconomico-culturel. Il offre ainsi l'opportunité de prendre en compte l'hétéronomie de cette population trop souvent envisagée de manière homogène. Cette cote sociale se décline en trois milieux socioculturels contrastés.

Tableau 1. Origine sociale : approche comparative

\begin{tabular}{|l|l|l|l|l|}
\hline & \multicolumn{2}{|l|}{ Apprentis } & \multicolumn{2}{l|}{ Lycéens } \\
\hline & Fréquence & $\%$ & Fréquence & $\%$ \\
\hline
\end{tabular}




\begin{tabular}{|l|l|l|l|l|}
\hline Milieux populaires & 244 & 55 & 64 & 55,5 \\
\hline Milieux intermédiaires & 53 & 13 & 22 & 13,5 \\
\hline Milieux favorisés & 76 & 17 & 28 & 16,5 \\
\hline Non réponses & 66 & 15 & 26 & 15,5 \\
\hline Total & 411 & 100 & 170 & 100,5 \\
\hline
\end{tabular}

Table 1. Social background: A comparative approach origine scolaire beaucoup plus diversifiée que celle des lycéens. Nous observons que $75 \%$ des lycéens viennent du collège et plus précisément $68 \%$ sont issus du palier d'orientation de la $3^{\mathrm{e}}$ générale. L'origine scolaire des apprentis est beaucoup plus hétérogène $\left(\mathrm{X}^{2}(5)=64.64, p<.0001\right) .38 \%$ seulement viennent d'une troisième générale. $50 \%$ ont une autre origine scolaire : $16 \%$ étaient notamment inscrits dans l'enseignement professionnel (lycée professionnel ou CFA) l'année précédente; d'autres ont suivi antérieurement un parcours en lycée général $(8 \%)$ ou dans la filière technologique (5\%) qui a pu, pour certains, les amener jusqu'au bac (8 apprentis titulaires d'un baccalauréat sur 43 soit $19 \%$, aucun chez les lycéens).

Bien que la majorité de notre population $(74,5 \%)$ ait redoublé au cours de la scolarité antérieure, il apparaît clairement que les apprentis sont significativement plus nombreux $(80 \%$ contre $61 \%)$ à avoir redoublé $(\mathrm{X} 2(1)=28.42, p<.0001)$. Plusieurs spécificités caractérisent ces redoublements :

Tableau 2. Première classe redoublée

\begin{tabular}{|c|c|c|c|c|c|}
\hline & CP-CE1 & $3^{e}$ cycle & $6^{e}$ & $5^{e}$ et + & Total \\
\hline \multicolumn{6}{|l|}{ Apprentis } \\
\hline Effectif & 107 & 72 & 79 & 80 & 338 \\
\hline$\%$ en ligne & $32 \%$ & $21 \%$ & $23 \%$ & $24 \%$ & $100 \%$ \\
\hline \multicolumn{6}{|l|}{ Lycéens } \\
\hline
\end{tabular}




\begin{tabular}{|c|c|c|c|c|c|}
\hline $\begin{array}{l}\text { Effectif } \\
\% \text { en ligne }\end{array}$ & $\begin{array}{l}23 \\
22 \%\end{array}$ & $\begin{array}{l}14 \\
14 \%\end{array}$ & \begin{tabular}{|l|}
24 \\
$24 \%$
\end{tabular} & $\begin{array}{l}40 \\
40 \%\end{array}$ & $\begin{array}{l}101 \\
100 \%\end{array}$ \\
\hline \multicolumn{6}{|l|}{ Total } \\
\hline Effectif & 130 & 86 & 103 & 120 & 439 \\
\hline$\%$ en ligne & $30 \%$ & $20 \%$ & $23 \%$ & $27 \%$ & $100 \%$ \\
\hline
\end{tabular}

Table 2. First repeated class

Les apprentis ont redoublé de manière plus précoce, dès l'école primaire pour $53 \%$ d'entre eux contre $36 \%$ des lycéens $\left(\mathrm{X}^{2}(3)=11.50, p<.01\right) .23 \%$ des apprentis redoublants et $18 \%$ des lycéens redoublants ont redoublé deux fois et plus $\left(X^{2}(1)=2.28\right.$, ns).

\section{Stratégie de recueil de données et démarches d'analyse}

17 Le recueil de données a été réalisé à partir de deux épreuves cognitives et un questionnaire.

Pour étudier l'influence du parcours scolaire antérieur sur le devenir des élèves au sein de la formation professionnelle initiale, nous avons souhaité prendre en compte le niveau scolaire des apprentis et lycéens. Nous avons volontairement écarté la possibilité de nous appuyer sur les notes obtenues dans le cursus antérieur. Si elles reflètent sans aucun doute un certain niveau de compétences, elles peuvent aussi être considérées comme très influencées par la capacité des jeunes concernés à se conformer aux attentes du système scolaire.

Deux épreuves ont été sélectionnées :

Une épreuve de raisonnement logique [test d'Aptitude Non Verbal de Naglieri (NNAT), 1996-1998]

Ce test " permet d'appréhender d'une façon culturellement équitable les aptitudes non verbales. Le NNAT est basé sur le principe des Matrices Analogiques" (Naglieri, 1998, 1). La passation de la forme $\mathrm{F}$ de l'épreuve (destinée aux collégiens de la $5^{\mathrm{e}}$ à la $3^{\mathrm{e}}$ ) s'effectue en trente minutes environ. Elle a été retenue en raison de sa relative indépendance par rapport au niveau socioculturel des sujets. Les situations de résolution mobilisent des formes géométriques et ne nécessitent pas une connaissance verbale particulière ou un vocabulaire spécifique. Cette épreuve peut également être utilisée auprès de jeunes dont la langue maternelle n'est pas le français ou pour ceux dont l'usage de la langue pose problème en général.

21 Les notes obtenues (dont le score maximum est égal à 38) ont été réparties en quatre niveaux, en fonction des effectifs, de façon à ce que chacun niveau regroupe $1 / 4$ de la population globale : Niveau $1:$ de 2 à 13 , Niveau $2:$ de 14 à 20 , Niveau $3:$ de 21 à 26 , Niveau 4 : de 27 à 37.

Une épreuve de langage écrit (d'une durée de 30 minutes environ) qui permet d'évaluer les capacités de compréhension et de production élémentaire L'épreuve consiste à lire un court texte lacunaire (mots manquants) et à s'engager dans une activité de complètement des lacunes (une lacune correspond à un mot) grâce aux inférences élaborées à partir de la compréhension du texte. Les notes de maitrise de l'écrit (dont le score maximum est de 42) ont également été réparties en quatre niveaux de la manière suivante : Niveau $1:$ de 1 à 22 , Niveau $2:$ de 23 à 28 , Niveau $3:$ de 29 à 32 , Niveau $4:$ de 33 et 39 . 

votre orientation (3 items) ? Avez-vous le sentiment que votre avis a compté dans votre orientation? Êtes-vous satisfait(e) de votre orientation? Votre père? Votre mère? Auriez-vous préféré une autre orientation ? Quel métier aimeriez-vous exercer plus tard ? Vous sentez-vous prêt(e) à exercer actuellement un métier? Qu'attendez-vous d'un métier? générale, vous apprenez pour : avoir un diplôme et trouver du travail ; aller plus loin dans vos études, faire plaisir à vos parents... (11 items) ? Vous êtes en apprentissage/lycée professionnel pour : apprendre un métier précis; apprendre autrement qu'au collège ; pour trouver plus facilement du travail... (11 items)? Pour vous, apprendre, c'est : comprendre et s'approprier des idées nouvelles; acquérir une culture générale; s'approprier des choses ennuyeuses... (16 items) ? Où, préférez-vous apprendre : au CFA/ lycée ; en entreprise ; chez vous? Que préférez-vous apprendre : les matières générales au CFA/ lycée; les savoirs présentés en entreprise; les matières professionnelles au CFA/ lycée? Quelles façons d'apprendre préférez-vous: lire; faire des travaux pratiques; discuter avec les enseignants ... (13 items) ? À l'école quelle était votre matière préférée ? À l'école quelle était la matière que vous aimiez le moins ?

31 - Informations sociobiographiques (11 questions) : cette rubrique permet de repérer la situation familiale de chaque jeune à partir d'une série de critères : son âge, son sexe, sa nationalité, celle de son père et de sa mère, la profession et le niveau d'étude de chacun de ses parents, la présence éventuelle d'artisans dans la famille.

Le recueil de données a été réalisé à l'entrée en formation, au début de la première année, deux mois environ après la rentrée scolaire. Les passations collectives du questionnaire, du test d'Aptitude Non Verbal de Naglieri (1998) et de l'épreuve de langage écrit (closure) 
ont eu lieu dans les établissements (lycées ou CFA), en classe sous la supervision d'un chercheur en deux séances.

Nous avons ensuite réévalué la situation des 641 jeunes en deux temps : pour recenser les abandons de formation, un an après la passation des premières épreuves soit entre les mois de septembre et novembre de la deuxième année ; pour évaluer la réussite au CAP au mois de juillet après la publication des résultats par le rectorat. Ce dispositif prospectif nous a permis d'étudier les caractéristiques spécifiques des sujets avant qu'ils soient confrontés à une situation de rupture ou qu'ils restent mobilisés dans le dispositif de formation jusqu'à la réussite de leur parcours de formation.

En ce qui concerne la stratégie d'analyse des résultats, différents types d'analyses statistiques (univariée de type $\mathrm{Khi}^{2}$ ou analyse de la variance) ont été utilisés en fonction de la nature des variables étudiées. Les questions relatives au rapport à l'apprendre ont fait l'objet d'une construction spécifique. Le rapport à l'apprendre est une notion complexe dont l'opérationnalisation dans un cadre extensif ne va pas de soi. Dans cette étude, nous nous sommes plus particulièrement intéressés au rapport épistémique (qu'est-ce qu'apprendre ?) et au rapport identitaire (pourquoi apprendre ?) à l'apprendre tels que définis par Charlot (1999).

À partir des réponses aux questions : "Pour vous, apprendre est un plaisir? ", "Pour vous apprendre, c'est?», «De façon générale, vous apprenez pour?", «Vous êtes en apprentissage/lycée pour? », nous avons regroupé à partir d'une démarche hypothético déductive différents items pour construire 4 indicateurs dont l'homogénéité respective est la suivante (alpha de Cronbach) :

Tableau 3. Typologie des formes de rapport à l'apprendre

\begin{tabular}{|c|c|}
\hline & $\begin{array}{l}\text { Le rapport épistémique } \\
\text { Qu'est-ce qu'apprendre? }\end{array}$ \\
\hline $\begin{array}{l}\text { Le rapport épistémique } \\
9 \text { items } \\
\alpha=.82 \\
\text { Renvoie à la conception/définition que le sujet donne à } \\
\text { l'acte d'apprendre }\end{array}$ & $\begin{array}{l}\text { Question } 22 \text { - Apprendre, c'est: } \\
1 \text { comprendre et s'approprier des } \\
\text { idées nouvelles } \\
2 \text { acquérir des théories abstraites } \\
3 \text { réfléchir, penser d'une autre } \\
\text { façon } \\
4 \text { acquérir des méthodes de travail } \\
5 \text { faire des choses avec ses mains, } \\
\text { acquérir des savoirs faire } \\
6 \text { acquérir une culture générale } \\
7 \text { acquérir une rigueur, une logique } \\
\text { dans le raisonnement } \\
8 \text { savoir refaire ce que quelqu'un } \\
\text { vient de vous montrer } \\
\text { Question } 32 \text { - Vous êtes en apprentissage } \\
\text { pour: } \\
9 \text { acquérir des connaissances } \\
\text { pratiques }\end{array}$ \\
\hline
\end{tabular}




\begin{tabular}{|c|c|}
\hline & $\begin{array}{l}\text { Le rapport identitaire } \\
\text { Pourquoi apprendre? }\end{array}$ \\
\hline $\begin{array}{l}\text { Apprendre pour soi, } \\
\text { par plaisir } \\
\text { (rapport réflexif) } \\
9 \text { items } \\
\alpha=.80 \\
\text { Renvoie aux mobiles de l'engagement dans l'acte } \\
\text { d'apprendre liés au développement personnel }\end{array}$ & $\begin{array}{l}\text { Question } 21 \text { - Pour vous : } \\
1 \text { apprendre est un plaisir } \\
\text { Question } 22 \text { - Apprendre, c'est : } \\
2 \text { satisfaire sa curiosité } \\
3 \text { savoir des choses qui permettent } \\
\text { de mieux se connaître soi-même } \\
4 \text { apprendre à vivre } \\
\text { Question } 24 \text { - Vous apprenez pour: } \\
5 \text { devenir autonome } \\
6 \text { vous faire plaisir } \\
7 \text { devenir adulte } \\
\text { Question } 32 \text { - Vous êtes en apprentissage } \\
\text { pour: } \\
8 \text { satisfaire une passion } \\
9 \text { devenir adulte, faire votre vie }\end{array}$ \\
\hline $\begin{array}{l}\text { Apprendre pour avoir un métier, par nécessité } \\
\text { (rapport utilitaire) } \\
8 \text { items } \\
\alpha=.83 \\
\text { Renvoie aux mobiles de l'engagement dans l'acte } \\
\text { d'apprendre fondés sur un abord instrumental des } \\
\text { études }\end{array}$ & $\begin{array}{l}\text { Question } 22 \text { - Apprendre, c'est: } \\
1 \text { assimiler les savoirs nécessaires } \\
\text { pour faire un métier précis } \\
2 \text { s'approprier des choses qui } \\
\text { servent dans la vie de tous les jours } \\
\text { Question } 24 \text { - Vous apprenez pour: } \\
3 \text { savoir des choses qui serviront } \\
\text { plus tard dans la vie de tous les jours } \\
4 \text { avoir un diplôme et trouver du } \\
\text { travail } \\
5 \text { être capable de faire un métier } \\
\text { précis } \\
6 \text { aller plus loin dans vos études } \\
7 \text { assimiler des bases qui servent } \\
\text { dans l'entreprise } \\
\text { Question } 32 \text { - Vous êtes en apprentissage } \\
\text { pour: } \\
8 \text { apprendre un métier précis } \\
9 \text { trouver plus facilement du travail } \\
10 \text { avoir un salaire }\end{array}$ \\
\hline
\end{tabular}




\begin{tabular}{|c|c|}
\hline $\begin{array}{l}\text { Apprendre pour les autres, par obligation } \\
\text { (rapport externalisé) } \\
8 \text { items } \\
\alpha=.62 \\
\text { Renvoie aux mobiles de l'engagement dans l'acte } \\
\text { d'apprendre vécus comme "imposés" ou comme un } \\
\text { choix par « défaut" }\end{array}$ & $\begin{array}{l}\text { Question } 22 \text { - Apprendre, c'est: } \\
1 \text { s'approprier des choses } \\
\text { ennuyeuses } \\
\text { Question } 24 \text { - Vous apprenez pour: } \\
2 \text { faire plaisir à vos parents } \\
3 \text { par obligation } \\
4 \text { vous n'avez pas de raison précise } \\
\text { Question } 32 \text { - Vous êtes en apprentissage } \\
\text { pour: } \\
5 \text { apprendre autrement qu'au } \\
\text { collège } \\
6 \text { ne pas rester sans rien faire } \\
7 \text { quitter l'école } \\
8 \text { vous n'avez pas de raison précise }\end{array}$ \\
\hline
\end{tabular}

Table 3. Typology of relationships to learning

Le premier indicateur concerne le rapport épistémique. Il a été plus particulièrement analysé à partir des réponses à la question "Pour vous apprendre, c'est? » qui propose 16 items visant à étudier le rapport des acteurs à un objet virtuel (le savoir) incarné dans des objets empiriques (par exemple les livres...). Il permet d'approcher la représentation que les sujets ont de la nature de cet objet (des idées nouvelles, des théories abstraites, des savoir-faire, etc.).

Les trois autres indicateurs se rapportent à l'étude du rapport identitaire. Chacun de ces indicateurs est non exclusif des autres. Ils offrent l'opportunité de repérer les mobiles qui animent les sujets selon qu'ils se fondent sur une dynamique plutôt centrée sur le développement personnel ou au contraire sur un rapport plus instrumental voire externalisé à l'apprendre.

L'ensemble de ces analyses nous a permis de repérer les facteurs susceptibles d'influencer la construction du parcours au sein de la formation professionnelle. Nous avons dans un deuxième temps réalisé une analyse discriminante de l'ensemble des dimensions significatives afin de repérer les plus influentes. Compte tenu des spécificités de chaque contexte de formation ainsi que de chacune des populations, la comparaison s'est effectuée intra-groupe (exemple: entre apprentis selon la rupture de contrat et la réussite au diplôme) afin de repérer les dimensions opérantes dans la construction du parcours au sein de chacun de ces deux secteurs de la formation professionnelle.

Cette étude offre ainsi l'opportunité d'étudier la diversité et les spécificités de l'expérience scolaire et du rapport à l'apprendre des élèves exclus de l'enseignement général au moment de leur sortie du système secondaire. Apprentis et lycéens ont-ils les mêmes curricula? Quel a été le parcours de ces jeunes avant leur entrée dans l'enseignement professionnel? Quelles sont leurs compétences cognitives, leur rapport à l'avenir? Quel sens donnent-ils à leur expérience scolaire? Quels savoirs et manières d'apprendre privilégient- ils? 


\section{La construction des parcours de formation au sein de la formation professionnelle initiale : conditions de réussite}

\section{Diversité et principales caractéristiques des parcours de formation}

Au terme de leurs deux années de formation, 75 \% des lycéens rencontrés au temps 1 (127 sur 170) ont obtenu leur diplôme contre 48 \% des apprentis (208 sur 434). Parmi les jeunes qui n'ont pas validé leur formation (44 lycéens soit $25 \%$ de l'effectif total et 226 apprentis soit $52 \%)$, deux trajectoires différentes se dessinent :

$41-50 \%$ des lycéens sans diplôme $(N=22)$ ont abandonné leur formation au cours de la première année et $50 \%$ ont échoué à l'examen. Ainsi, si on tient compte uniquement des lycéens présents à l'examen $(N=148)$, le taux d'échec au CAP/BEP concerne $11,5 \%$ de la population totale pour un taux d'abandon global de $12,9 \%$.

- $59 \%$ des apprentis sans certification $(N=133)$ ont rompu un contrat d'apprentissage au cours de la première année et $41 \%$ ont véritablement échoué à l'examen. Ainsi, si on tient compte uniquement des apprentis présents à l'examen $(N=303)$, le taux d'échec au CAP/ BEP se réduit de $48 \%$ à $31 \%(N=93)$ soit $21 \%$ de la population totale $(N=434)$, ce qui reste néanmoins nettement supérieur à celui des lycéens. Parmi eux, nous retrouvons 37 apprentis qui ont rompu un contrat d'apprentissage, ce qui porte le taux global d'abandon véritable de la formation à $29,7 \%$ (133 apprentis concernés). Il s'agit de jeunes qui ont signé un nouveau contrat d'apprentissage avec une nouvelle entreprise après que nous ayons évalué le nombre de rupture(s) au début de leur $2 e$ année de formation et qui sont parvenus au terme de leur formation. On repère ici que la rupture de contrat d'apprentissage n'aboutit pas systématiquement à une sortie de système de formation sans diplôme puisque au final, en moyenne, $22 \%$ de la totalité des apprentis en rupture (1 et $2 \mathrm{ou}+)$ obtiennent leur CAP $\left(\mathrm{X}^{2}(2)=76.44, p<.0001\right)$.

\section{Les facteurs déterminants de la trajectoire : approche comparative}

Tableau 4. Les facteurs de l'abandon : approche comparative

\begin{tabular}{l} 
Lycéens « décrocheurs " \\
Abandon $: N=22$ \\
Pas d'abandon $: N=148$ \\
\hline Variables significatives
\end{tabular} \begin{tabular}{l|l|l|l|}
$\mathrm{Khi}^{2}$ & ou \\
$\mathrm{F}^{*}$ & ddl & $p$
\end{tabular}




\begin{tabular}{|c|c|c|c|}
\hline $\begin{array}{l}\text { Parcours scolaire antérieur } \\
\text { - ont redoublé dans leur cursus antérieur } \\
\text { Compétences cognitives } \\
\text { - maîtrisent moins bien l'écrit que leurs homologues } \\
\text { Expérience scolaire } \\
\text { - estiment avoir eu de moins bons résultats scolaires au collège que } \\
\text { leurs } \\
\text { homologues } \\
\text { - déclarent plus souvent que les autres ne pas aimer aller à l'école } \\
\text { - ont une image d'eux-mêmes en tant qu'élève plus dévalorisée } \\
\text { - ont une préférence pour une autre orientation } \\
\text { Rapport à l'apprendre } \\
\text { - disent plus souvent ne pas approuver de plaisir dans l'acte } \\
\text { d'apprendre } \\
\text { - donnent un sens à l'acte d'apprendre et à leur formation qui s'inscrit } \\
\text { moins } \\
\text { que les autres dans les registres : } \\
\text { - utilitaire } \\
\text { - identitaire } \\
\text { - épistémique }\end{array}$ & $\begin{array}{c}6,75^{*} \\
4,38^{*} \\
3,95^{*} \\
3.68^{*} \\
3,88^{*} \\
5,97^{*} \\
5,18^{*} \\
7,34^{*} \\
7,27^{*} \\
13,06^{*}\end{array}$ & $\begin{array}{l}1 \\
1 \\
1 \\
1 \\
1 \\
1 \\
1 \\
1 \\
1 \\
1\end{array}$ & $\begin{array}{l}.01 \\
.05 \\
.05 \\
.05 \\
.05 \\
.01 \\
.05 \\
.01 \\
.01 \\
.0001\end{array}$ \\
\hline \multicolumn{4}{|l|}{$\begin{array}{l}\text { Apprentis qui ont rompu leur contrat } \\
1 \text { rupture }: N=141 \\
2 \text { ruptures et }+: N=29 \\
\text { Pas de rupture }: N=271\end{array}$} \\
\hline Variables significatives & $\begin{array}{ll}\mathrm{Khi}^{2} & \text { ou } \\
\mathrm{F}^{*} & \end{array}$ & ddl & $p$ \\
\hline $\begin{array}{l}\text { Expérience scolaire } \\
\text { - le fait d'avoir manqué volontairement les cours } \\
\text { - une représentation du métier d'élève très dévalorisée }\end{array}$ & & 2 & $\begin{array}{l}.05 \\
.01\end{array}$ \\
\hline
\end{tabular}

Table 4. Dropout factors: A comparative approach

43 Pour identifier les facteurs susceptibles d'influencer l'abandon et/ou la réussite au diplôme au sein des deux populations, nous avons réalisé des analyses univariées qui nous ont permis d'identifier plusieurs spécificités. La rupture de contrat d'apprentissage ne relève pas de la même dynamique que l'abandon en lycée professionnel.

Genèse des ruptures de contrat d'apprentissage Contrairement aux lycéens, il existe peu de différences entre les apprentis en rupture et leurs homologues. Nos résultats montrent que les caractéristiques du parcours scolaire antérieur, les compétences cognitives, le rapport à l'apprendre et le sens de l'orientation jouent un rôle tout à fait négligeable dans la genèse des ruptures de contrat. 

un modèle permettant de classer $88 \%$ (150 sur 170) des observations à partir de 4 dimensions :

\section{Alternance et réussite au CAP}

Tableau 5. Les facteurs de la réussite au diplôme : approche comparative 


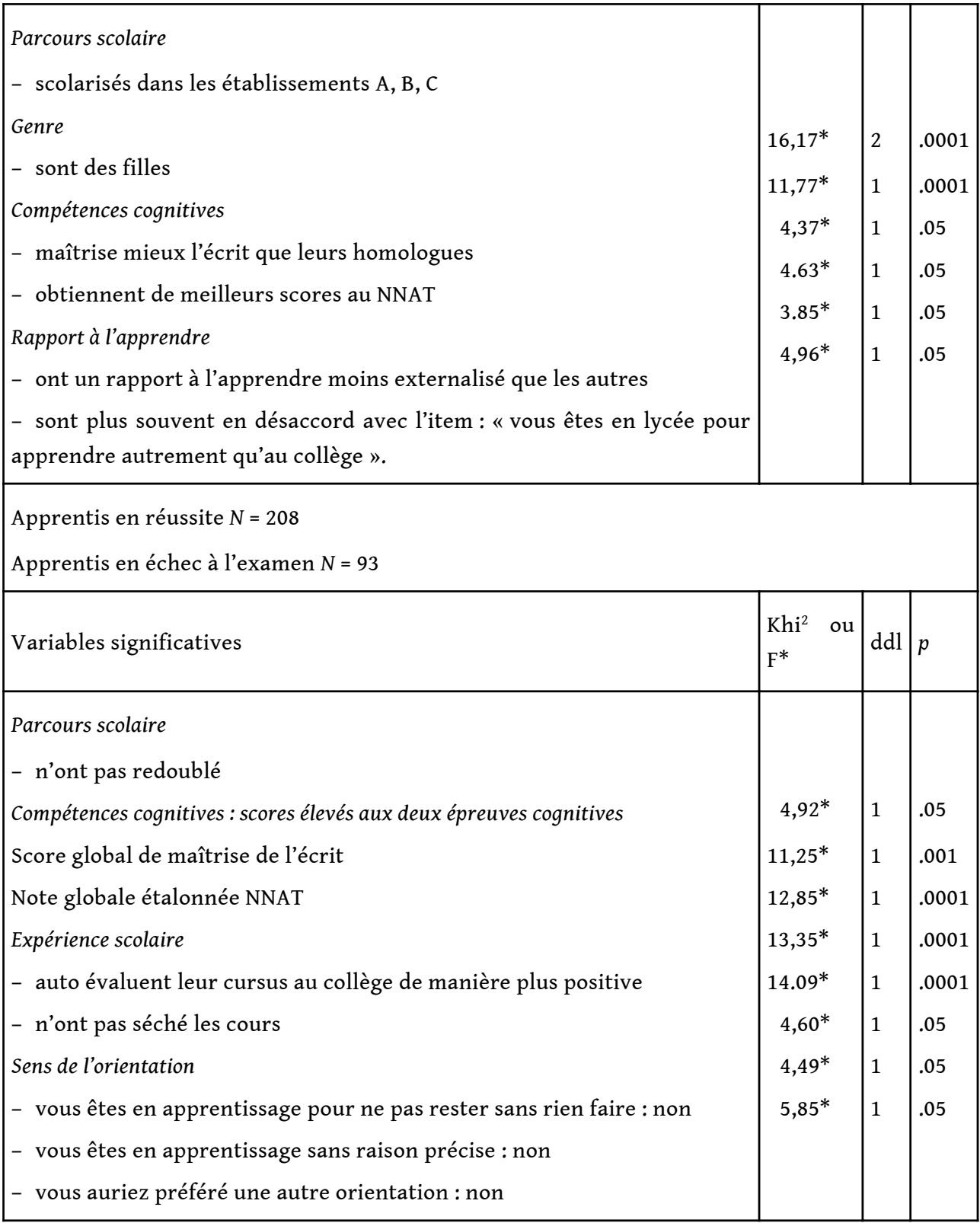

Table 5. Success factors for achieving diploma: A comparative approach

61 Nos analyses révèlent que les apprentis qui réussissent à leur examen sont plus souvent des apprentis qui :

62 - n'ont pas redoublé ;

63 - ont des scores élevés aux deux épreuves cognitives ;

64 - auto-évaluent leur cursus au collège de manière plus positive ;

65 - n'ont pas séché les cours ;

66 - ne sont pas en apprentissage pour ne pas rester sans rien faire ;

67 - ne sont pas en apprentissage sans raison précise ;

68 - n'auraient pas préféré une autre orientation. 
69 Le modèle issu de l'analyse discriminante [Lambda de Wilks $=0,904 ; \mathrm{Khi}^{2}=20.21 ; p$ $<.0001 ; \mathrm{ddl}=3$ ] réalisée sur ces 8 facteurs statistiquement significatifs isole 3 dimensions très différenciatrices des deux groupes d'apprentis permettant de classer $63 \%$ des observations :

70 - avoir une auto-évaluation positive des résultats obtenus au collège (.70) ;

71 - avoir un score global de maîtrise de l'écrit élevé (.58) ;

72 - ne pas avoir de préférence pour une autre orientation (1.48).

73 Comme pour les lycéens, la réussite des apprentis apparaît très corrélée au sens de l'orientation et aux acquis du cursus antérieur. Le niveau scolaire est très corrélé à la réussite au CAP. À condition qu'ils poursuivent leur formation jusqu'à son terme, les apprentis qui ont les niveaux de compétences à l'écrit les plus élevés sont les plus assurés d'obtenir leur diplôme.

\section{Les conditions de la réussite en lycée professionnel}

74 Le groupe des lycéens qui ont obtenu leur CAP/BEP présente un certain nombre de spécificités.

75 Il s'agit plutôt de jeunes :

76 - scolarisés dans les établissements A ou C ;

77 - qui sont des filles;

78 - qui maîtrisent mieux l'écrit que leurs homologues ;

79 - qui obtiennent de meilleurs scores au NNAT $^{5}$ ont un rapport à l'apprendre moins externalisé que les autres ;

80 - sont plus souvent en désaccord avec l'item: «vous êtes en lycée pour apprendre autrement qu'au collège ».

81 L'analyse discriminante [Lambda de Wilks $=0.891 ; \mathrm{Khi}^{2}=16.23 ; p<.0001 ; \mathrm{ddl}=2$ ] fondée sur la prise en compte de $67 \%$ des observations $(n=144)$ indique que deux dimensions exercent un rôle prépondérant:

82 - être une fille (.82) ;

83 - obtenir un score élevé au NNAT (.58).

84 Ainsi contrairement au phénomène de l'abandon qui dépend avant tout du rapport à l'apprendre, il semble que la réussite au diplôme dépende avant tout du niveau des compétences logiques des candidats au CAP/ BEP.

\section{Discussion}

Comment lutter contre les sorties du système éducatif sans qualification des élèves de la formation professionnelle initiale? Nos analyses montrent qu'il n'est pas possible d'envisager les mêmes leviers d'interventions pour les apprentis et les lycéens professionnels. L'arrêt prématuré de la formation aussi bien que l'échec à l'examen relève de dynamiques très différentes au sein et entre les deux systèmes de formation.

86 Conformément à notre hypothèse, l'abandon en lycée professionnel, plus circonscrit que chez les apprentis ( $13 \%$ de l'échantillon contre $30 \%$ pour les apprentis) apparait très 
corrélé aux formes de rapport au savoir construites dans le cadre du parcours scolaire antérieur. Il s'étaye sur une dynamique où le rapport à l'apprendre et le sens de l'expérience scolaire jouent un rôle important. Contrairement à ce qui se passe pour les apprentis, il est possible d'identifier un groupe de lycéens qui développe un rapport épistémique à l'apprendre très externalisé. Plus en difficulté sur le plan scolaire, ils ont souvent redoublé, ont du mal à donner un sens à leur orientation et sont peu mobilisés par les apprentissages scolaires. Dans leur cas, il semble qu'une intervention précoce visant à permettre un travail réflexif sur le sens de l'orientation en lien avec l'histoire scolaire et familiale et non pas uniquement la construction d'un projet professionnel puisse offrir l'opportunité d'éviter les effets néfastes d'un rapport à la forme scolaire (Lahire, 1995 ; Vincent, 1980) qui était déjà difficile au collège.

À la différence de ce qui se passe pour les lycéens et à l'encontre de notre hypothèse, la genèse des ruptures de contrat d'apprentissage peut difficilement être expliquée à partir du versant psychologique de la différenciation sociale et des processus qui ont modelé la construction du parcours antérieur. Nos analyses ne permettent pas d'identifier véritablement une dimension liée à la rupture ultérieure. Pour les apprentis, seule la réussite à l'examen apparait influencée par des éléments en lien avec la trajectoire antérieure (plus particulièrement le niveau de compétences à l'écrit). Ainsi, il n'existe pas au regard des critères pris en compte dans cette recherche d'apprentis à risque de rupture. À l'encontre de tous les stéréotypes véhiculés à ce propos, ces résultats permettent de conclure que la rupture de contrat d'apprentissage ne peut être ramenée à une simple erreur d'orientation ou à des difficultés d'adaptation (ACFCI, 2011). Qu'ils aient un projet explicite ou non, que celui ci-soit cohérent ou non avec leur formation, les apprentis sont tous susceptibles de rompre leur contrat d'apprentissage. La rupture de contrat d'apprentissage constitue un phénomène généralisé susceptible de concerner tous les apprentis quels que soient leur niveau de compétences cognitives et les caractéristiques de leur parcours dans le secondaire. Seul le phénomène de la multirupture, très minoritaire (29 apprentis, $7 \%$ de l'échantillon), semble corrélé à des profils spécifiques où les apprentis en souffrance apparaissent très en difficulté sur le plan de la socialisation et développent un rapport à l'avenir difficile (de Léonardis, CapdevielleMougnibas, \& Prêteur, 2006).

Ces résultats viennent ainsi étayer les constats réalisés par Cart, Henguelle et ToutinTrelcat (2010), Capdevielle-Mougnibas et de Léonardis (2010) ou encore Lamamra et Masdonati (2009) qui identifient comme principale cause de rupture les caractéristiques de la socialisation professionnelle mise en œuvre au sein des entreprises. Contrairement à ce qui se passe pour les lycéens, l'entrée en apprentissage tend à réduire l'importance des acquis de la période antérieure, offrant sans aucun doute l'occasion d'une nouvelle forme de mobilisation sur les savoirs proposés. Ainsi, contrairement aux préconisations formulées dans la plupart des rapports visant la prise en charge des ruptures de contrat, la préparation de l'orientation ne constitue pas un levier suffisant pour espérer lutter contre ce phénomène. Au contraire, si l'on tient compte des études citées ci-dessus, il semble que le défaut d'organisation pédagogique du cadre de formation offert par l'entreprise et le CFA, les formes de dominations dont l'apprenti peut faire l'objet au sein de l'entreprise et du CFA ainsi que le manque ou l'absence de valorisation de l'identité professionnelle de ses apprentis semblent être les dimensions sur lesquelles il paraît beaucoup plus intéressant d'agir pour contribuer à réduire le nombre de ces arrêts anticipés de formation. 
89 Au terme de nos analyses, cette étude extensive apporte une contribution à l'étude du monde de la formation professionnelle initiale de niveau $\mathrm{V}$. Comme en échos aux récentes préconisations politiques qui voient dans la filière de l'apprentissage salarié un moyen de lutter contre l'échec et le décrochage scolaire en permettant aux jeunes qui ne sont pas à l'aise dans l'organisation pédagogique classique du collège de trouver une voie de formation qui leur convienne, nos résultats montrent en effet que la réussite des apprentis en CFA est moins tributaire des aléas du parcours et de l'expérience scolaire antérieure que celles de leurs homologues en lycée professionnel. Mis en perspective avec les bons résultats des filières de l'apprentissage en matière d'insertion professionnelle, ils pourraient donner à penser que l'entrée dans l'apprentissage est un moyen d'offrir une nouvelle chance à ces jeunes souvent en échec, d'assurer leur avenir professionnel. C'est oublier néanmoins que l'apprentissage ne constitue pas une garantie de réussite absolue. Nos observations le montrent: au final $75 \%$ des lycéens rencontrés contre $48 \%$ des apprentis ont obtenu leur diplôme au bout des deux ans de leur formation. Qui plus est, la réussite au diplôme des apprentis comme celle des lycéens, apparaît très tributaire de leurs niveaux de compétences logiques et de maîtrise de l'écrit. Dans cette perspective, il semble qu'une entrée anticipée en apprentissage telle que préconisée depuis 2011 dans le cadre de la mise en place du nouveau Dispositif d'Initiation aux Métiers de l'Alternance (DIMA) ou encore de la mise en place de la classe de troisième de découverte professionnelle (3e DP6), qui prépare une orientation vers le lycée professionnel, puisse à l'encontre des objectifs annoncés, contribuer à favoriser l'échec des publics les plus en difficulté, si elle néglige le fait que l'acquisition d'un niveau scolaire minimum constitue un préalable incontournable pour la réussite au diplôme au sein des deux filières de la formation professionnelle.

Dans l'état actuel de l'organisation du système de formation par apprentissage, il n'est pas possible de se contenter de postuler que la qualité de la préparation de l'orientation et du projet professionnel du futur apprenti ou encore le contact avec le monde du travail puisse à lui seul suffire à permettre à ces jeunes de retrouver une motivation et à investir leur formation. Ce pari apparaît on ne peut plus risqué, tant il occulte les enjeux identitaires de la période du développement où se trouvent ces jeunes et les difficultés posées par la mission de formation qui incombe à l'entreprise dans le cadre d'un contrat d'apprentissage. L'entrée en apprentissage constitue une épreuve pour tous ces jeunes, épreuve dans laquelle ils n'ont pas d'autres choix que d'engager leur identité et leur histoire. Ils ne pourront la traverser qu'à la condition de trouver des partenaires capables d'entendre leur désir d'apprendre autrement qu'à l'école et de créer des conditions de formation qui constituent un socle sur lesquels ils pourront s'appuyer pour se construire.

\section{BIBLIOGRAPHIE}

ACFCI (2011). Les ruptures de contrats d'apprentissage... une fatalité ? (Analyse et commentaires du réseau des CCI, 4 p). http://www.centre-inffo.fr/uhfp/webographie2012/pdf/acfci_hd-1.pdf (page consultée le 3 mars 2012). 
Broccolichi, S., \& Largueze, B. (1996). Les sorties sans qualification moins de cinq ans après l'entrée au collège. Éducation et Formations, 48, 81-102.

Caille, J.-P. (2000). Qui sort sans qualification du système éducatif ? Éducation et Formations, 57, 1937.

Capdevielle-Mougnibas,V., Prêteur, Y., \& Rossi-Neves, P. (2008, 16 et 17 juin). Diversité et paradoxes des curricula des élèves d'origine populaire exclus de l'enseignement général. L'exemple des apprentis et lycéens professionnels de niveau $V$. Communication présentée au colloque international de Sociologie de l'éducation Ce que l'école fait aux individus. Centre de Recherche en Éducation de Nantes, Nantes, France. http://www. cren-nantes.net/IMG/pdf/CapdeviellePreteur Rossi.pdf (page consultée le 3 mars 2012).

Capdevielle-Mougnibas, V. (2010). Sens de l'expérience scolaire, socialisation différenciée et orientation vers la formation professionnelle initiale de niveau V. In V. Rouyer, S. Croity-Belz \& Y. Prêteur (éd.). Genre et socialisation de l'enfance à l'adolescence. Expliquer les différences. Penser l'égalité (pp. 85- 97). Ramonville-Saint-Agne, Éditions Erès.

Capdevielle-Mougnibas, V., \& de Léonardis, M. (2010). Ségrégation sociale et responsabilité du chercheur en psychologie : donner la parole à ceux qui ne l'ont pas. Recherche Qualitative, 29(2), 134-159.

Capdevielle-Mougnibas, V., Garric, N., Courtinat-Camps, A., \& Favreau, C. (2012). Diversité des formes du rapport au savoir chez des lycéen(ne)s professionnels et apprenti(e)s de niveau V. Neuropsychiatrie de l'Enfance et de l'Adolescence, 60(2), 94-100.

Cart, B., Henguelle, V., \& Toutin-Trelcat, M.-H. (2010). Contrat d'apprentissage, les raisons de la rupture. BrefCereq, 272, 1-4.

Charlot, B. (1999). Le rapport au savoir en milieu populaire. Une recherche dans les lycées professionnels de banlieue. Paris : Anthropos.

Charlot, B., Emin, L., \& Jellab, A. (2002). L'abandon scolaire en cours de formation : le cas des élèves de BEP. Commissions professionnelles consultatives documents, 2002/7, 1-100.

Courtinat-Camps, A., \& Prêteur, Y. (2012). Diversité des expériences scolaires chez des collégien (ne)s scolarisés en troisième. L'Orientation Scolaire et Professionnelle, 41(4).

de Léonardis, M., Capdevielle-Mougnibas, V., \& Prêteur, Y., (2006). Sens de l'orientation vers l'apprentissage des apprentis de niveau $\mathrm{V}$ : entre expérience scolaire et rapport à l'avenir. L'Orientation Scolaire et Professionnelle, 35(1), 5-27.

Esterle-Hedibel, M. (2007). Les élèves transparents. Les arrêts de scolarité avant 16 ans. Villeneuved'Ascq : Presses Universitaires du Septentrion.

Favreau, C., \& Capdevielle-Mougnibas, V. (2011). Formation par alternance : expérience scolaire et rapport à l'apprendre chez des apprentis de niveau $\mathrm{V}$ et leurs maîtres d'apprentissage. Psychologie du travail et des organisations, 17(2), 253-268.

Fortin, L., Royer, É., Potvin, P., Marcotte, D., \& Yergeau, E., (2004). La prédiction du risque de décrochage scolaire au secondaire : facteurs personnels, familiaux et scolaires. Revue canadienne des sciences du comportement, 36(3), 219-231.

Gagné, M.-E., \& Marcotte, D., (2010), Effet médiateur de l'expérience scolaire sur la relation entre la dépression et le risque de décrochage scolaire chez les adolescents vivant la transition primaire-secondaire, Revue de psycho-éducation, 39(1), 27-44. 
Guba, E.-G., \& Lincoln, Y.-S. (1994). Competing paradigms in qualitative research. In N. K. Denzin \& Y. S. Lincoln (Eds.), Handbook of qualitative research (pp. 105-117). London: Sage.

Joseph, O., Lopez, A., \& Ryk, F. (2008). Génération 2004, des jeunes pénalisés par la conjoncture. BrefCereq, 248, 1-4.

Lahire, B. (1995). Tableaux de familles : heurs et malheurs scolaires en milieux populaires. Paris, Gallimard/Seuil, collection « Hautes Études ».

Lamamra, N., \& Masdonati, J. (2009). Arrêter une formation professionnelle. Lausanne : Antipodes.

Langevin, L. (1999). L'abandon scolaire. Outremont (Québec) : Éditions Logiques (2e édition).

Langouët, G. (2003). Les oubliés de l'école en France. Paris : Hachette.

Laterrasse, C., \& Brossais, E. (2006). Du rapport au savoir. In J. Beillerot \& N. Mosconi (éd.), Traité des sciences et des pratiques de l'éducation (pp. 381-393). Paris : Dunod.

Léger, F. (2008). Les sorties sans qualification : la baisse se poursuit. Note d'information, 08-05. http://media.education.gouv.fr/file/2008/41/4/ni0805_22414.pdf (page consultée le 3 mars 2012).

Lessard, A., Fortin, L., Joly, J., Royer, E., Marcotte, D., \& Potvin, P. (2007). Cheminement de décrocheurs et de décrocheuses, Revue des sciences de l'éducation, 33(3), 647- 662.

Malrieu, P. (1978). Psychologies génétiques et psychologie historique. Journal de Psychologie, 3 , 261-277.

Malrieu, P. (1995). La personnalisation : analyse psycho-sociale. Aprendizagem/Desenvolvimento, 4, 15-16.

Malrieu, P. (2003). Le sens des dires autobiographiques. Toulouse : Erès.

MEN-DEPP (2008). L'État de l'école. Édition 2007. Paris : MEN- DEPP. http://

media.education.gouv.fr/file/etat18/17/0/

etat18_41170.pdf (page consultée le 3 mars 2012).

Naglieri, J.-A. (1998). Test d'aptitude non verbale de Naglieri (NNAT). Paris : ECPA.

Rochex, J.-Y. (1995). Le sens de l'expérience scolaire. Paris : Presses Universitaires de France.

Rochex, J.-Y. (1998). Rapport au savoir, activité intellectuelle et élaboration de soi : du malentendu au décrochage ? In M.-C. Bloch \& B. Gerde, Association La Bouture, Les lycéens décrocheurs. De l'impasse aux chemins de traverse (pp. 131-141). Lyon : Chronique sociale.

Vincent, G. (1980). L'école primaire française : étude sociologique. Lyon : PUL.

Von Glasersfeld, E. (1990). An exposition of constructivism: Why some like it radical. In R. B. Davis, C. A. Maher \& N. Noddings (Eds.), Constructivism views on the teaching and learning of mathematics (pp. 19-29). Reston, Virginia: National Council of Teachers of Mathematics.

\section{NOTES}

1. Les chiffres les plus récents témoignent cependant d'une légère baisse des effectifs entre 2001 et 2005. Auparavant, la France a connu deux périodes de diminution importante de ce phénomène : au cours des années soixante-dix et durant les années quatre-vingt. Au total, en trente ans, le nombre de sortants sans qualification a été divisé par quatre : la part des élèves 
déscolarisés de façon précoce est passée de $25 \%$ à $6 \%$ au sein d'une génération entre 1975 et 2005 (Léger, 2008).

2. Différentes formes d'abandon peuvent être envisagées: quitter effectivement le système scolaire mais aussi s'absenter régulièrement, sélectionner les cours ou encore être peu mobilisés sur les activités scolaires (Charlot et al., 2002).

3. Recherche financée par la Chambre Régionale de Métiers et de l'Artisanat et le Conseil Régional de la Région Midi-Pyrénées ainsi que le PIREF ACI Éducation et Formation.

4. Même si on observe quelques trajectoires atypiques (par exemple, deux lycéennes préparent un CAP/ BEP en mécanique, une autre un CAP/BEP en plomberie / deux apprenties et quatre lycéennes préparent un CAP/BEP peinture, etc.), plus de $85 \%$ des filles de notre échantillon préparent un CAP/BEP coiffure ou esthétique. La question du genre, prise en compte dans cette étude donne lieu à des résultats spécifiques mais ne sera pas abordée dans le cadre de cette contribution.

5. Rappelons que le NNAT est une épreuve de raisonnement logique [test d'Aptitude Non Verbal de Naglieri (NNAT)], 1996-1998.

\section{RÉSUMÉS}

En France, les abandons de formation et l'échec au diplôme des apprentis et lycéens professionnels de niveau $\mathrm{V}$ représente $60 \%$ des sorties du système éducatif. Une recherche longitudinale, auprès de 611 sujets, montre que la genèse de ces deux phénomènes relève de dynamiques très différentes au sein et entre les deux systèmes de formation. Alors que le devenir des lycéens apparait corrélé à un rapport au savoir construit au cours du parcours scolaire antérieur, la rupture de contrat et l'échec à l'examen des apprentis peuvent difficilement être expliqués à partir d'un rapport à l'apprendre et d'un sens de l'expérience scolaire spécifiques.

In France, V-level vocational secondary school students and apprentices who quit their training programmes and fail to achieve their diplomas make up $60 \%$ of all dropouts from the educational system. This longitudinal research study of 611 participants showed that the origins of these two phenomena reveal strikingly different dynamics within and between these two training systems. While the future of the secondary school students appeared correlated with the relationship to knowledge which they developed along their previous academic path, the apprentices' breach of contract and failure to achieve their diploma could not be so easily explained by any specific relationship to knowledge or sense of academic experience.

\section{INDEX}

Mots-clés : diplôme de niveau $\mathrm{V}$, formation professionnelle initiale, rapport au savoir, rupture de contrat d'apprentissage, sorties du système scolaire sans qualification

Keywords : breaches of apprenticeship contracts, Initial vocational training, Level V diploma, Relationship to knowledge, Educational system dropouts 


\section{AUTEURS}

\section{VALÉRIE CAPDEVIELLE-MOUGNIBAS}

est Maître de Conférences de psychologie clinique, Université de Toulouse le Mirail - Thèmes de recherche : rapport au savoir, conditions de réussite et construction des parcours de formation dans l'apprentissage salarié - Contact : Université de Toulouse le Mirail, Laboratoire Psychologie du développement et processus de socialisation (EA 1687), Maison de la Recherche, Université Toulouse le Mirail. 5 allées Antonio Machado, Toulouse 31 058, Cedex 9 - Courriel : capdevie@univ-tlse2.fr

\section{YVES PRÊTEUR}

est Professeur de psychologie du développement et de l'éducation - Thèmes de recherche : éducation familiale et scolaire, expérience scolaire et construction identitaire à l'adolescence Contact : Laboratoire Psychologie du développement et processus de socialisation (EA 1687), Maison de la Recherche, Université Toulouse le Mirail. 5 allées Antonio Machado, Toulouse 31 058, Cedex 9, France - Courriel : ypreteur@univ-tlse2.fr

\section{CÉCILE FAVREAU}

est Doctorante, Chargée d'études (Convention CIFRE) - Thèmes de recherche : rapport au savoir, pratiques tutorales et rapport à la fonction de tuteur des artisans maitres d'apprentissage Contact : Laboratoire Psychologie du développement et processus de socialisation (EA 1687) et Chambre régionale de Métiers et de l'Artisanat de Midi-Pyrénées (CRMA). 59 ter Chemin Verdale, 31240 Saint-Jean - Courriel : cecilefavreau@gmail.com 